\title{
On fractional differential equations and inclusions with nonlocal and average-valued (integral) boundary conditions
}

\author{
Bashir Ahmad ${ }^{1 *}$, Sotiris K Ntouyas ${ }^{1,2}$, Ravi P Agarwal ${ }^{3}$ and Ahmed Alsaedi ${ }^{1}$
}

\author{
"Correspondence: \\ bashirahmad_qau@yahoo.com \\ 'Nonlinear Analysis and Applied \\ Mathematics - Research Group \\ (NAAM), King Abdulaziz University, \\ P.O. Box 80203, Jeddah, 21589, Saudi \\ Arabia \\ Full list of author information is \\ available at the end of the article
}

\begin{abstract}
This paper is concerned with the existence of solutions for boundary value problems of fractional differential equations and inclusions supplemented with nonlocal and average-valued (integral) boundary conditions. The existence results for the single-valued case (equations) are obtained by means of fixed point theorems due to O'Regan and Sadovski, whereas the existence of solutions for the multivalued case (inclusions) is established via nonlinear alternative for contractive maps. The obtained results are well illustrated by examples.
\end{abstract}

MSC: 34A08; 34B10; 34A60

Keywords: Caputo fractional derivative; equations; inclusions; nonlocal and integral boundary conditions; fixed point

\section{Introduction}

The study of fractional differential equations has recently attracted the attention of many researchers and modelers. The interest in the subject owes to its widespread applications in a variety of applied sciences and engineering disciplines such as biological sciences, ecology, aerodynamics, control theory, viscoelasticity, electro-dynamics of complex medium, electron-analytical chemistry, environmental issues, et cetera. The recent trend in the mathematical modeling of several phenomena indicates the popularity of fractional calculus modeling tools due to the nonlocal characteristic of fractional-order differential and integral operators, which are capable of tracing the past history of many materials and processes; see, for instance, [1-7] and the references therein.

Differential inclusions, regarded as a generalization of differential equations and inequalities, have very important and interesting applications in optimal control theory and stochastic processes [8]. In fact, the tools of differential inclusions facilitate the investigation of dynamical systems having velocities not uniquely determined by the state of the system.

Boundary value problems of fractional-order differential equations and inclusions supplemented with several kinds of conditions such as classical, nonlocal, multipoint, periodic/antiperiodic, fractional-order, and integral boundary conditions have extensively been investigated by many researchers. In particular, the study of nonlocal boundary value problems finds interesting applications in physical and chemical processes, where the

(c) 2016 Ahmad et al. This article is distributed under the terms of the Creative Commons Attribution 4.0 International License (http://creativecommons.org/licenses/by/4.0/), which permits unrestricted use, distribution, and reproduction in any medium, provided you give appropriate credit to the original author(s) and the source, provide a link to the Creative Commons license, and indicate if changes were made. 
classical initial/boundary conditions fail to describe some peculiar phenomena occurring inside the domain. On the other hand, integral boundary conditions help to formulate computational fluid dynamics (blood flow) problems in a better way as such conditions allow one to describe the cross-section of vessels in a more realistic arbitrary manner instead of always assuming circular type cross-section [9]. Also, ill-posed parabolic backward problems in time partial differential equations can be regularized with the aid of integral boundary conditions; see, for example, mathematical models for bacterial selfregularization [10]. For details and examples, we refer the reader to a variety of results [11-26]. In a recent article [27], the authors studied a boundary value problem of fractional differential equations with nonlocal and average-type integral boundary conditions given by

$$
\left\{\begin{array}{l}
{ }^{\mathrm{c}} D^{\alpha} x(t)=f\left(t, x(t),{ }^{\mathrm{c}} D^{\beta} x(t)\right), \quad 0<t<1, \\
x(0)+x^{\prime}(0)=h(x), \quad \int_{0}^{\eta} x(t) d t=\xi, \quad 0<\eta<1, \xi \in \mathbb{R}, \\
x^{\prime \prime}(0)=x^{\prime \prime \prime}(0)=\cdots=x^{(n-1)}(0)=0,
\end{array}\right.
$$

where ${ }^{\mathrm{c}} D^{\alpha}$ and ${ }^{\mathrm{c}} D^{\beta}$ denote the Caputo fractional derivatives of orders $\alpha$ and $\beta$ with $n-$ $1<\alpha<n(n \geq 2)$ and $0<\beta<1$, and $f:[0,1] \times \mathbb{R} \times \mathbb{R} \rightarrow \mathbb{R}$ and $h: C([0,1], \mathbb{R}) \rightarrow \mathbb{R}$ are continuous functions. Applying the Leray-Schauder nonlinear alternative, Krasnoselskii's fixed point theorem and Banach's fixed point theorem together with Hölder inequality, some existence results for problem (1.1) were obtained.

The objective of the present paper is to continue the study initiated in [27] and provide a variety in the existence criteria for solutions of the problem at hand. Precisely, we establish two more existence results for problem (1.1), which are based on fixed point theorems due to O'Regan and Sadovski (Section 3). Then we switch onto investigating the multivalued analogue of (1.1)

$$
\left\{\begin{array}{l}
{ }^{\mathrm{c}} D^{\alpha} x(t) \in F\left(t, x(t),{ }^{\mathrm{c}} D^{\beta} x(t)\right), \quad 0<t<1, \\
x(0)+x^{\prime}(0)=h(x), \quad \int_{0}^{\eta} x(t) d t=\xi, \quad 0<\eta<1, \\
x^{\prime \prime}(0)=x^{\prime \prime \prime}(0)=\cdots=x^{(n-1)}(0)=0,
\end{array}\right.
$$

where $F:[0,1] \times \mathbb{R} \times \mathbb{R} \rightarrow \mathcal{P}(\mathbb{R})$ is a multivalued map, and $\mathcal{P}(\mathbb{R})$ is the family of all nonempty subsets of $\mathbb{R}$. In Section 4 , we discuss the existence of solutions for problem (1.2) by means of the nonlinear alternative for contractive maps.

\section{Preliminaries}

In this section, we present some basic definitions on fractional calculus and an auxiliary lemma $[1,3]$.

Definition 2.1 The fractional integral of order $r$ with the lower limit zero for a function $f$ is defined as

$$
I^{r} f(t)=\frac{1}{\Gamma(r)} \int_{0}^{t} \frac{f(s)}{(t-s)^{1-r}} d s, \quad t>0, r>0,
$$

provided that the right-hand side is pointwise defined on $[0, \infty)$, where $\Gamma$ is the gamma function defined by $\Gamma(r)=\int_{0}^{\infty} t^{r-1} e^{-t} d t$. 
Definition 2.2 The Riemann-Liouville fractional derivative of order $r>0, n-1<r<n$, $n \in N$, of a function $f$ is defined as

$$
D_{0+}^{r} f(t)=\frac{1}{\Gamma(n-r)}\left(\frac{d}{d t}\right)^{n} \int_{0}^{t}(t-s)^{n-r-1} f(s) d s
$$

provided that the function $f$ has absolutely continuous derivatives up to order $(n-1)$.

Definition 2.3 The Caputo derivative of order $r$ of a function $f:[0, \infty) \rightarrow R$ is defined as

$$
{ }^{\mathrm{c}} D^{r} f(t)=D^{r}\left(f(t)-\sum_{k=0}^{n-1} \frac{t^{k}}{k !} f^{(k)}(0)\right), \quad t>0, n-1<r<n .
$$

Remark 2.1 If $f(t) \in C^{n}[0, \infty)$, then

$$
{ }^{\mathrm{c}} D^{r} f(t)=\frac{1}{\Gamma(n-r)} \int_{0}^{t} \frac{f^{(n)}(s)}{(t-s)^{r+1-n}} d s=I^{n-r} f^{(n)}(t), \quad t>0, n-1<q<n .
$$

We need the following known lemma [27].

Lemma 2.1 Let $y \in A C[0,1]$ and $x \in A C^{n}[0,1]$. Then the linear problem

$$
\left\{\begin{array}{l}
{ }^{\mathrm{c}} D^{\alpha} x(t)=y(t), \quad 0<t<1, \\
x(0)+x^{\prime}(0)=h(x), \quad \int_{0}^{\eta} x(t) d t=\xi, \quad 0<\eta<1, \\
x^{\prime \prime}(0)=x^{\prime \prime \prime}(0)=\cdots=x^{(n-1)}(0)=0,
\end{array}\right.
$$

is equivalent to the fractional integral equation

$$
\begin{aligned}
x(t)= & \int_{0}^{t} \frac{(t-s)^{\alpha-1}}{\Gamma(\alpha)} y(s) d s+\frac{2(1-t)}{\eta(2-\eta)} \xi+\frac{2 t-\eta}{2-\eta} h(x) \\
& +\frac{2(t-1)}{\eta(2-\eta)} \int_{0}^{\eta} \frac{(\eta-s)^{\alpha}}{\Gamma(\alpha+1)} y(s) d s .
\end{aligned}
$$

\section{Existence results for a single-valued problem}

Define the space $X=\left\{x \mid x \in C([0,1], \mathbb{R})\right.$ and $\left.{ }^{\mathrm{c}} D^{\beta} x \in C([0,1], \mathbb{R})\right\}$ equipped with the norm $\|x\|_{X}=\|x\|+\left\|{ }^{\mathrm{c}} D^{\beta} x\right\|=\sup _{t \in[0,1]}|x(t)|+\sup _{t \in[0,1]}\left|{ }^{\mathrm{c}} D^{\beta} x(t)\right|$. Clearly, $\left(X,\|\cdot\|_{X}\right)$ is a Banach space [28].

In view of Lemma 2.1, we introduce the operator $F: X \rightarrow X$ by

$$
\begin{aligned}
F(x)(t)= & \int_{0}^{t} \frac{(t-s)^{\alpha-1}}{\Gamma(\alpha)} f\left(s, x(s),{ }^{c} D^{\beta} x(s)\right) d s+\frac{2(1-t)}{\eta(2-\eta)} \xi+\frac{2 t-\eta}{2-\eta} h(x) \\
& +\frac{2(t-1)}{\eta(2-\eta)} \int_{0}^{\eta} \frac{(\eta-s)^{\alpha}}{\Gamma(\alpha+1)} f\left(s, x(s),{ }^{c} D^{\beta} x(s)\right) d s,
\end{aligned}
$$

which can be expressed as

$$
(F x)(t)=\left(F_{1} x\right)(t)+\left(F_{2} x\right)(t), \quad t \in[0,1],
$$


where $F_{1,2}: X \rightarrow X$ are given by

$$
\begin{aligned}
\left(F_{1} x\right)(t)= & \int_{0}^{t} \frac{(t-s)^{\alpha-1}}{\Gamma(\alpha)} f\left(s, x(s),{ }^{\mathrm{c}} D^{\beta} x(s)\right) d s \\
& +\frac{2(t-1)}{\eta(2-\eta)} \int_{0}^{\eta} \frac{(\eta-s)^{\alpha}}{\Gamma(\alpha+1)} f\left(s, x(s),{ }^{\mathrm{c}} D^{\beta} x(s)\right) d s, \\
\left(F_{2} x\right)(t)= & \frac{2(1-t)}{\eta(2-\eta)} \xi+\frac{2 t-\eta}{2-\eta} h(x) .
\end{aligned}
$$

For computational convenience, we set the notations

$$
\Lambda_{1}:=\frac{1}{\Gamma(\alpha+1)}+\frac{2}{\eta \Gamma(\alpha+2)}+\frac{1}{\Gamma(\alpha-\beta+1)}+\frac{2}{\eta \Gamma(2-\beta)} \frac{1}{\Gamma(\alpha+2)}
$$

and

$$
\Lambda_{2}:=\frac{2|\xi|}{\eta}\left(1+\frac{1}{\Gamma(2-\beta)}\right), \quad \Lambda_{3}=2+\eta+\frac{2}{\Gamma(2-\beta)} .
$$

Our first existence result relies on a fixed point theorem of O'Regan [29].

Lemma 3.1 Denote by $U$ an open set in a closed convex set $C$ of a Banach space E. Assume that $0 \in U$. Also assume that $F(\bar{U})$ is bounded and that $F: \bar{U} \rightarrow C$ is given by $F=F_{1}+F_{2}$, where $F_{1}: \bar{U} \rightarrow E$ is continuous and completely continuous, and $F_{2}: \bar{U} \rightarrow E$ is a nonlinear contraction (i.e., there exists a nonnegative nondecreasing function $\phi:[0, \infty) \rightarrow[0, \infty)$ such that $\phi(z)<z$ for $z>0$ and $\left\|F_{2}(x)-F_{2}(y)\right\| \leq \phi(\|x-y\|)$ for all $\left.x, y \in \bar{U}\right)$. Then, either

(C1) F has a fixed point $u \in \bar{U}$; or

(C2) there exist a point $u \in \partial U$ and $\lambda \in(0,1)$ with $u=\lambda F(u)$, where $\bar{U}$ and $\partial U$, respectively, represent the closure and boundary of $U$.

In the proof of the next result, we use the notations

$$
\Omega_{r}=\left\{x \in X:\|x\|_{X}<r\right\}
$$

and

$$
M_{r}=\max \{|f(t, x, y)|:(t, x) \in[0,1] \times[-r, r] \times[-r, r]\} .
$$

Theorem 3.1 Assume that:

$\left(\mathrm{A}_{1}\right) \quad h: C([0,1], \mathbb{R}) \rightarrow \mathbb{R}$ is a continuous function satisfying the condition

$$
|h(u)-h(v)| \leq \ell\|u-v\|, \quad \forall u, v \in C([0,1], \mathbb{R}),
$$

with $\ell>0$ such that $\ell \Lambda_{3}<1$, where $\Lambda_{3}$ is given by (3.5);

$\left(\mathrm{A}_{2}\right) h(0)=0$;

$\left(\mathrm{A}_{3}\right)$ there exist a nonnegative function $p \in C([0,1], \mathbb{R})$ and nondecreasing functions $\psi_{i}$ : $\mathbb{R}^{+} \rightarrow \mathbb{R}^{+}, i=1,2$, such that

$$
|f(t, x, y)| \leq p(t)\left(\psi_{1}(|x|)+\psi_{2}(|y|)\right)
$$

for all $(t, x, y) \in[0,1] \times \mathbb{R} \times \mathbb{R}$ 
$\left(\mathrm{A}_{4}\right) \sup _{r \in(0, \infty)} \frac{r}{\Lambda_{1}\|p\|\left[\psi_{1}(r)+\psi_{2}(r)\right]+\Lambda_{2}}>\frac{1}{1-\ell \Lambda_{3}}$, where $\Lambda_{1}$ is given by (3.4) and $\Lambda_{i}, i=2,3$, are defined by (3.5) (note that $\ell \Lambda_{3}<1$ by $\left(\mathrm{A}_{1}\right)$ ).

Then the boundary value problem (1.1) has at least one solution on $[0,1]$.

Proof By assumption $\left(\mathrm{A}_{4}\right)$, there exists a number $r_{0}>0$ such that

$$
\frac{r_{0}}{\Lambda_{1}\|p\|\left[\psi_{1}\left(r_{0}\right)+\psi_{2}\left(r_{0}\right)\right]+\Lambda_{2}}>\frac{1}{1-\ell \Lambda_{3}} .
$$

We shall show that the operators $F_{1}$ and $F_{2}$ defined by (3.2) and (3.3), respectively, satisfy all the conditions of Lemma 3.1.

Step 1 . The operator $F_{1}$ is completely continuous. We first show that $F_{1}\left(\bar{\Omega}_{r_{0}}\right)$ is bounded. For any $x \in \bar{\Omega}_{r_{0}}$, we have

$$
\begin{aligned}
\left\|F_{1} x\right\| \leq & \int_{0}^{t} \frac{(t-s)^{\alpha-1}}{\Gamma(\alpha)}\left|f\left(s, x(s),{ }^{\mathrm{c}} D^{\beta} x(s)\right)\right| d s \\
& +\frac{2(t-1)}{\eta(2-\eta)} \int_{0}^{\eta} \frac{(\eta-s)^{\alpha}}{\Gamma(\alpha+1)}\left|f\left(s, x(s),{ }^{\mathrm{c}} D^{\beta} x(s)\right)\right| d s \\
\leq & \int_{0}^{t} \frac{(t-s)^{\alpha-1}}{\Gamma(\alpha)}\left|f\left(s, x(s),{ }^{\mathrm{c}} D^{\beta} x(s)\right)\right| d s \\
& +\frac{2(t-1)}{\eta(2-\eta)} \int_{0}^{1} \frac{(1-s)^{\alpha}}{\Gamma(\alpha+1)}\left|f\left(s, x(s),{ }^{\mathrm{c}} D^{\beta} x(s)\right)\right| d s \\
\leq & M_{r}\left[\frac{1}{\Gamma(\alpha+1)}+\frac{2}{\eta \Gamma(\alpha+2)}\right]
\end{aligned}
$$

and

$$
\begin{aligned}
\left\|{ }^{c} D^{\beta}\left(F_{1} x\right)\right\| & \leq M_{r} \int_{0}^{t} \frac{(t-s)^{\alpha-1-\beta}}{\Gamma(\alpha-\beta)} d s+M_{r} \frac{2 t^{1-\beta}}{\eta \Gamma(2-\beta)} \int_{0}^{\eta} \frac{(\eta-s)^{\alpha}}{\Gamma(\alpha+1)} d s \\
& \leq M_{r} \int_{0}^{t} \frac{(t-s)^{\alpha-1-\beta}}{\Gamma(\alpha-\beta)} d s+M_{r} \frac{2}{\eta \Gamma(2-\beta)} \int_{0}^{1} \frac{(1-s)^{\alpha}}{\Gamma(\alpha+1)} d s \\
& \leq M_{r}\left\{\frac{1}{\Gamma(\alpha-\beta+1)}+\frac{2}{\eta \Gamma(2-\beta)} \frac{1}{\Gamma(\alpha+2)}\right\} .
\end{aligned}
$$

Consequently, we have

$$
\begin{aligned}
\left\|F_{1} x\right\|_{X} & =\left\|F_{1} x\right\|+\left\|{ }^{\mathrm{c}} D^{\beta}\left(F_{1} x\right)\right\| \\
& \leq M_{r}\left\{\frac{1}{\Gamma(\alpha+1)}+\frac{2}{\eta \Gamma(\alpha+2)}+\frac{1}{\Gamma(\alpha-\beta+1)}+\frac{2}{\eta \Gamma(2-\beta)} \frac{1}{\Gamma(\alpha+2)}\right\},
\end{aligned}
$$

which shows that the operator $F_{1}\left(\bar{\Omega}_{r_{0}}\right)$ is uniformly bounded.

Further, for $0 \leq t_{1}<t_{2} \leq 1$, we have

$$
\begin{aligned}
\left|\left(F_{1} x\right)\left(t_{2}\right)-\left(F_{1} x\right)\left(t_{1}\right)\right| \leq & \mid \frac{1}{\Gamma(\alpha)} \int_{0}^{t_{1}}\left[\left(t_{2}-s\right)^{\alpha-1}-\left(t_{1}-s\right)^{\alpha-1}\right] f\left(s, x(s),{ }^{\mathrm{c}} D^{\beta} x(s)\right) d s \\
& +\frac{1}{\Gamma(\alpha)} \int_{t_{1}}^{t_{2}}\left(t_{2}-s\right)^{\alpha-1} f\left(s, x(s),{ }^{\mathrm{c}} D^{\beta} x(t)\right) d s \mid
\end{aligned}
$$




$$
\begin{aligned}
& +\frac{2\left(t_{2}-t_{1}\right)}{\eta(2-\eta)} \int_{0}^{\eta} \frac{(\eta-s)^{\alpha}}{\Gamma(\alpha+1)}\left|f\left(s, x(s),{ }^{\mathrm{c}} D^{\beta} x(s)\right)\right| d s \\
\leq & \frac{M_{r}}{\Gamma(\alpha+1)}\left(t_{2}^{\alpha}-t_{1}^{\alpha}\right)+\frac{2 M_{r}\left(t_{2}-t_{1}\right)}{\eta \Gamma(\alpha+2)}
\end{aligned}
$$

and

$$
\begin{aligned}
\left|{ }^{\mathrm{c}} D^{\beta}\left(F_{1} x\right)\left(t_{2}\right)-{ }^{\mathrm{c}} D^{\beta}\left(F_{1} x\right)\left(t_{1}\right)\right| \leq & \frac{M_{r}}{\Gamma(\alpha-\beta+1)}\left(t_{2}^{\alpha-\beta-1}-t_{1}^{\alpha-\beta-1}\right) \\
& +\frac{2 M_{r}}{\eta \Gamma(\alpha+2) \Gamma(2-\beta)}\left(t_{2}^{1-\beta}-t_{1}^{1-\beta}\right) .
\end{aligned}
$$

Obviously, $\left\|\left(F_{1} x\right)\left(t_{2}\right)-\left(F_{1} x\right)\left(t_{1}\right)\right\|_{X} \rightarrow 0$ as $t_{2} \rightarrow t_{1}$. Hence, the operator $F_{1}$ is equicontinuous. Thus, it follows by the Arzelá-Ascoli theorem that $F_{1}\left(\bar{\Omega}_{r_{0}}\right)$ is relatively compact.

Now, let $x_{n} \subset \bar{\Omega}_{r_{0}}$ with $\left\|x_{n}-x\right\|_{X} \rightarrow 0$. Then the limit $\left\|x_{n}(t)-x(t)\right\| \rightarrow 0$ uniformly on $[0,1]$. From the uniform continuity of $f(t, x, y)$ on the compact set $[0,1] \times\left[-r_{0}, r_{0}\right] \times\left[-r_{0}, r_{0}\right]$ it follows that $\left\|f\left(t, x_{n}(t),{ }^{\mathrm{c}} D^{\beta} x_{n}(t)\right)-f\left(t, x(t),{ }^{\mathrm{c}} D^{\beta} x(t)\right)\right\| \rightarrow 0$ uniformly on [0,1]. Hence, $\left\|F_{1} x_{n}-F_{1} x\right\|_{X} \rightarrow 0$ as $n \rightarrow \infty$, which proves the continuity of $F_{1}$. This completes the proof of Step 1.

Step 2. The operator $F_{2}: \bar{\Omega}_{r_{0}} \rightarrow X$ is contractive. This is a consequence of $\left(\mathrm{A}_{2}\right)$. Indeed, for $x, y \in X$, we have

$$
\begin{aligned}
\left|\left(F_{2} x\right)(t)-\left(F_{2} y\right)(t)\right| & =\left|\frac{2 t-\eta}{2-\eta}\right||h(x)-h(y)| \\
& \leq(2+\eta)|h(x)-h(y)| \\
& \leq(2+\eta) \ell\|x-y\|,
\end{aligned}
$$

which, by taking the supremum for $t \in[0,1]$, yields

$$
\left\|\left(F_{2} x\right)-\left(F_{2} y\right)\right\| \leq(2+\eta) \ell\|x-y\| .
$$

Also, we have

$$
\left\|{ }^{\mathrm{c}} D^{\beta} F_{2} x-{ }^{\mathrm{c}} D^{\beta} F_{2} y\right\| \leq \frac{2}{\Gamma(2-\beta)} \ell\|x-y\| .
$$

Consequently, we get

$$
\left\|F_{2} x-F_{2} y\right\|_{X} \leq\left(2+\eta+\frac{2}{\Gamma(2-\beta)}\right) \ell\|x-y\|_{X},
$$

which, in view of $\left(\mathrm{A}_{1}\right)$, implies that $F_{2}$ is a contraction.

Step 3. The set $F\left(\bar{\Omega}_{r_{0}}\right)$ is bounded. Assumptions $\left(\mathrm{A}_{1}\right)$ and $\left(\mathrm{A}_{2}\right)$ imply that

$$
\begin{aligned}
\left|\left(F_{2} x\right)(t)\right| & \leq \frac{2(1-t)}{\eta(2-\eta)}|\xi|+\frac{|2 t-\eta|}{2-\eta}|h(x)| \\
& \leq \frac{2}{\eta}|\xi|+(2+\eta)(|h(x)-h(0)|+|h(0)|)
\end{aligned}
$$




$$
\begin{aligned}
& \leq \frac{2}{\eta}|\xi|+(2+\eta) \ell\|x\| \\
& \leq \frac{2}{\eta}|\xi|+(2+\eta) \ell r_{0}
\end{aligned}
$$

and

$$
\left|{ }^{\mathrm{c}} D^{\beta}\left(F_{2} x\right)(t)\right| \leq \frac{1}{\Gamma(2-\beta)}\left(\frac{2|\xi|}{\eta}+2 \ell r_{0}\right)
$$

Hence,

$$
\left\|F_{2} x\right\|_{X} \leq \frac{2|\xi|}{\eta}\left(1+\frac{1}{\Gamma(2-\beta)}\right)+\ell r_{0}\left(2+\eta+\frac{2}{\Gamma(2-\beta)}\right)
$$

for any $x \in \bar{\Omega}_{r_{0}}$. This, together with the boundedness of the set $F_{1}\left(\bar{\Omega}_{r_{0}}\right)$, implies that the set $F\left(\bar{\Omega}_{r_{0}}\right)$ is bounded.

Step 4. Finally, we will show that case (C2) in Lemma 3.1 does not hold. On the contrary, suppose that (C2) holds. Then, we have that there exist $\lambda \in(0,1)$ and $x \in \partial \Omega_{r_{0}}$ such that $x=\lambda F x$. So, we have $\|x\|_{X}=r_{0}$ and

$$
\begin{aligned}
x(t)= & \lambda \int_{0}^{t} \frac{(t-s)^{\alpha-1}}{\Gamma(\alpha)} f\left(s, x(s),{ }^{\mathrm{c}} D^{\beta} x(s)\right) d s+\lambda \frac{2(1-t)}{\eta(2-\eta)} \xi+\lambda \frac{2 t-\eta}{2-\eta} h(x) \\
& +\lambda \frac{2(t-1)}{\eta(2-\eta)} \int_{0}^{\eta} \frac{(\eta-s)^{\alpha}}{\Gamma(\alpha+1)} f\left(s, x(s),{ }^{\mathrm{c}} D^{\beta} x(s)\right) d s .
\end{aligned}
$$

Further, we have that

$$
\begin{aligned}
& |x(t)| \leq \int_{0}^{t} \frac{(t-s)^{\alpha-1}}{\Gamma(\alpha)} p(s)\left(\psi_{1}(|x(s)|)+\psi_{2}\left(\left|{ }^{\mathrm{c}} D^{\beta} x(s)\right|\right)\right) d s+\frac{2}{\eta}|\xi|+(2+\eta) \ell\|x\| \\
& +\frac{2}{\eta} \int_{0}^{\eta} \frac{(\eta-s)^{\alpha}}{\Gamma(\alpha+1)} p(s)\left(\psi_{1}(|x(s)|)+\psi_{2}\left(\left|{ }^{\mathrm{c}} D^{\beta} x(s)\right|\right)\right) d s \\
& \leq \int_{0}^{1} \frac{(1-s)^{\alpha-1}}{\Gamma(\alpha)} p(s)\left(\psi_{1}\left(\|x\|_{X}\right)+\psi_{2}\left(\|x\|_{X}\right)\right) d s+\frac{2}{\eta}|\xi|+(2+\eta) \ell\|x\|_{X} \\
& +\frac{2}{\eta} \int_{0}^{1} \frac{(1-s)^{\alpha}}{\Gamma(\alpha+1)} p(s)\left(\psi_{1}\left(\|x\|_{X}\right)+\psi_{2}\left(\|x\|_{X}\right)\right) d s \\
& \leq\|p\|\left[\psi_{1}\left(r_{0}\right)+\psi_{2}\left(r_{0}\right)\right]\left\{\frac{1}{\Gamma(\alpha+1)}+\frac{2}{\eta \Gamma(\alpha+2)}\right\}+\frac{2}{\eta}|\xi|+(2+\eta) \ell r_{0}, \\
& \left|{ }^{\mathrm{c}} D^{\beta} x(t)\right| \leq \int_{0}^{t} \frac{(t-s)^{\alpha-1-\beta}}{\Gamma(\alpha-\beta)} p(s)\left(\psi_{1}(|x|)+\psi_{2}\left(\left|{ }^{\mathrm{c}} D^{\beta} x(s)\right|\right)\right) d s \\
& +\frac{t^{1-\beta}}{\Gamma(2-\beta)} \frac{2}{2-\eta}\left|h(x)-\frac{\xi}{\eta}\right| \\
& +\frac{2}{\eta(2-\eta)} \frac{t^{1-\beta}}{\Gamma(2-\beta)} \int_{0}^{\eta} \frac{(\eta-s)^{\alpha}}{\Gamma(\alpha+1)} p(s)\left(\psi_{1}(|x|)+\psi_{2}\left(\left|{ }^{c} D^{\beta} x(s)\right|\right)\right) d s \\
& \leq \int_{0}^{t} \frac{(t-s)^{\alpha-1-\beta}}{\Gamma(\alpha-\beta)} p(s)\left(\psi_{1}\left(\|x\|_{X}\right)+\psi_{2}\left(\|x\|_{X}\right)\right) d s+\frac{2}{\Gamma(2-\beta)}\left(\ell\|x\|_{X}+\frac{|\xi|}{\eta}\right) \\
& +\frac{2}{\eta \Gamma(2-\beta)} \int_{0}^{1} \frac{(1-s)^{\alpha}}{\Gamma(\alpha+1)} p(s)\left(\psi_{1}\left(\|x\|_{X}\right)+\psi_{2}\left(\|x\|_{X}\right)\right) d s
\end{aligned}
$$




$$
\begin{aligned}
\leq & \|p\|\left[\psi_{1}\left(r_{0}\right)+\psi_{2}\left(r_{0}\right)\right]\left\{\frac{1}{\Gamma(\alpha-\beta+1)}+\frac{2}{\eta \Gamma(2-\beta)} \frac{1}{\Gamma(\alpha+2)}\right\} \\
& +\frac{2}{\Gamma(2-\beta)}\left(\ell r_{0}+\frac{|\xi|}{\eta}\right) .
\end{aligned}
$$

Thus, we have

$$
\begin{aligned}
r_{0} \leq & \|p\|\left[\psi_{1}\left(r_{0}\right)+\psi_{2}\left(r_{0}\right)\right]\left\{\frac{1}{\Gamma(\alpha+1)}+\frac{2}{\eta \Gamma(\alpha+2)}+\frac{1}{\Gamma(\alpha-\beta+1)}\right. \\
& \left.+\frac{2}{\eta \Gamma(2-\beta)} \frac{1}{\Gamma(\alpha+2)}\right\}+\frac{2|\xi|}{\eta}\left(1+\frac{1}{\Gamma(2-\beta)}\right)+\ell r_{0}\left(2+\eta+\frac{2}{\Gamma(2-\beta)}\right) \\
= & \Lambda_{1}\|p\|\left[\psi_{1}\left(r_{0}\right)+\psi_{2}\left(r_{0}\right)\right]+\Lambda_{2}+\ell \Lambda_{3} r_{0},
\end{aligned}
$$

which yields a contradiction,

$$
\frac{r_{0}}{\Lambda_{1}\|p\|\left[\psi_{1}\left(r_{0}\right)+\psi_{2}\left(r_{0}\right)\right]+\Lambda_{2}} \leq \frac{1}{1-\ell \Lambda_{3}} .
$$

Thus, the operators $F_{1}$ and $F_{2}$ satisfy all the conditions of Lemma 3.1. Hence, the operator $F$ has at least one fixed point $x \in \bar{\Omega}_{r_{0}}$, which is the solution of problem (1.1). This completes the proof.

Our second existence result is based on Sadovskii's fixed point theorem. Let us first recall some auxiliary material before proceeding further.

Definition 3.1 Let $M$ be a bounded set in metric space $(X, d)$. The Kuratowskii measure of noncompactness $\alpha(M)$ is defined as $\inf \{\epsilon: M$ covered by a finitely many sets such that the diameter of each set $\leq \epsilon\}$.

Definition 3.2 [30] Let $\Phi: \mathcal{D}(\Phi) \subseteq X \rightarrow X$ be a bounded and continuous operator on Banach space $X$. Then $\Phi$ is called a condensing map if $\alpha(\Phi(B))<\alpha(B)$ for all bounded sets $B \subset \mathcal{D}(\Phi)$, where $\alpha$ denotes the Kuratowski measure of noncompactness.

Lemma 3.2 ([31], Example 11.7) The map $K+C$ is a $k$-set contraction with $0 \leq k<1$, and thus also condensing, if

(i) $K, C: \mathcal{D} \subseteq X \rightarrow X$ are operators on the Banach space $X$;

(ii) $K$ is $k$-contractive, that is,

$$
\|K x-K y\| \leq k\|x-y\|
$$

for all $x, y \in \mathcal{D}$ and fixed $k \in[0,1)$;

(iii) $C$ is compact.

Lemma 3.3 [32] Let $B$ be a convex, bounded, and closed subset of a Banach space $X$, and $\Phi: B \rightarrow B$ be a condensing map. Then $\Phi$ has a fixed point.

Theorem 3.2 Assume that $\left(\mathrm{A}_{1}\right),\left(\mathrm{A}_{2}\right)$, and $\left(\mathrm{A}_{3}\right)$ hold. Then problem (1.1) has at least one solution on $[0,1]$. 
Proof Let $B_{r}=\left\{x \in X:\|x\|_{\leq} r\right\}$ be a closed bounded and convex subset of $X$, where $r$ will be fixed later. We define the map $F: B_{r} \rightarrow X$ by

$$
(F x)(t)=\left(F_{1} x\right)(t)+\left(F_{2} x\right)(t), \quad t \in[0,1],
$$

where $F_{1}$ and $F_{2}$ are defined by (3.2) and (3.3), respectively. Notice that problem (1.1) is equivalent to the fixed point problem $F(x)=x$.

Step 1. $(F x) B_{r} \subset B_{r}$.

For that, select $r \geq \frac{\Lambda_{1}\|p\|\left[\psi_{1}(r)+\psi_{2}(r)\right]+\Lambda_{2}}{1-\ell \Lambda_{3}}$, where $\Lambda_{i}, i=1,2,3$, are defined by (3.4) and (3.5). As in Theorem 3.1, Step 1, we can prove that

$$
\left\|F_{1} x\right\|_{X} \leq \Lambda_{1}\|p\|\left[\psi_{1}(r)+\psi_{2}(r)\right]
$$

and, as in Step 3, we can get

$$
\left\|F_{2} x\right\| \leq \Lambda_{2}+\ell r \Lambda_{3}
$$

Consequently,

$$
\|F x\|_{X}=\|F x\|+\left\|{ }^{c} D^{\beta}(F x)\right\| \leq \Lambda_{1}\|p\|\left[\psi_{1}(r)+\psi_{2}(r)\right]+\Lambda_{2}+\ell r \Lambda_{3}<r
$$

which implies that $(F x)\left(B_{r}\right) \subset B_{r}$.

Step 2. $F_{1}$ is compact.

This was proved in Theorem 3.1, Step 1.

Step 3. $F_{2}$ is continuous and $\gamma$-contractive.

To show the continuity of $F_{2}$ for $t \in[0,1]$, let us consider a sequence $x_{n}$ converging to $x$. Then, as in Step 2 of the proof of Theorem 3.1, we can show that

$$
\left\|F_{2} x_{n}-F_{2} x\right\|_{X} \leq\left(2+\eta+\frac{2}{\Gamma(2-\beta)}\right) \ell\left\|x_{n}-x\right\|
$$

which implies that $F_{2}$ is continuous. Also, $F_{2}$ is $\gamma$-contractive by Theorem 3.1 (Step 2) with $\gamma=\left(2+\eta+\frac{2}{\Gamma(2-\beta)}\right) \ell<1$.

Step $4 . F$ is condensing.

Since $T_{2}$ is a continuous $\gamma$-contraction and $T_{1}$ is compact, by Lemma 3.2, $F: B_{r} \rightarrow B_{r}$ with $F=F_{1}+F_{2}$ is a condensing map on $B_{r}$.

From the previous four steps we conclude by Lemma 3.3 that the map $F$ has a fixed point, which, in turn, implies that problem (1.1) has a solution.

Example 3.1 Consider the following boundary value problem:

$$
\begin{aligned}
& { }^{\mathrm{c}} D^{5 / 2} x(t)=\frac{e^{-2 t}}{\sqrt{9+t}}\left(a \sin x(t)+b \frac{{ }^{\mathrm{c}} D^{3 / 4} x(t)}{1+{ }^{\mathrm{c}} D^{3 / 4} x(t)}+\frac{1}{3}\right), \quad 0<t<1, \\
& x(0)+x^{\prime}(0)=\frac{1}{10} x(4 / 5), \quad x^{\prime \prime}(0)=0, \quad \int_{0}^{2 / 3} x(t) d t=\frac{1}{2},
\end{aligned}
$$

where $h(x)=\frac{1}{10} x(4 / 5), \eta=2 / 3, \xi=1 / 2$, and $a$ and $b$ are suitably chosen real numbers. 
Observe that

$$
\begin{aligned}
\left|f\left(t, x(t),{ }^{\mathrm{c}} D^{3 / 4} x(t)\right)\right| & =\left|\frac{e^{-2 t}}{\sqrt{9+t}}\left(a \sin x(t)+b \frac{{ }^{\mathrm{c}} D^{3 / 4} x(t)}{1+{ }^{\mathrm{c}} D^{3 / 4} x(t)}+\frac{1}{3}\right)\right| \\
& \leq p(t)\left(\psi_{1}(|x|)+\psi_{2}(|y|)\right)
\end{aligned}
$$

with $p(t)=e^{-2 t}, \psi_{1}(|x|)=a|x|, \psi_{2}\left(\left|{ }^{c} D^{3 / 4} x(t)\right|\right)=b+1 / 3$, and $\ell=1 / 10$ since

$$
|h(u)-h(v)| \leq \frac{1}{10}\|u-v\| .
$$

With the given values, we find that $\Lambda_{1} \simeq 1.199538, \Lambda_{2} \simeq 3.154894, \Lambda_{3} \simeq 3.769929$. Further, $\ell<(2+\eta+2 / \Gamma(2-\beta))^{-1}$ holds since $(2+\eta+2 / \Gamma(2-\beta))^{-1} \simeq 0.205204>\frac{1}{10}=\ell$, and $\left(\mathrm{A}_{4}\right)$ is satisfied for $0<a<1 / 2$ and for any finite real value of $b$ since $\frac{1}{1-\ell \Lambda_{3}} \simeq 1.605118$, and $\sup _{r \in(0, \infty)} \frac{r}{\Lambda_{1}\|p\|\left[\psi_{1}(r)+\psi_{2}(r)\right]+\Lambda_{2}} \simeq \frac{1}{1.199538 a}$. Thus, all the conditions of Theorem 3.1 are satisfied, and consequently there exists one solution for problem (3.7)-(3.8) on $[0,1]$.

Remark 3.1 Notice that Example 3.1 also illustrates Theorem 3.2 since assumptions $\left(A_{1}\right)$, $\left(\mathrm{A}_{2}\right)$, and $\left(\mathrm{A}_{3}\right)$ clearly hold.

\section{Existence results for multivalued problem (1.2)}

Let us recall some basic definitions on multivalued maps [33, 34].

For a normed space $(X,\|\cdot\|)$, let $P_{\mathrm{cl}}(X)=\{Y \in F(X): Y$ is closed $\}, P_{\mathrm{b}}(X)=\{Y \in$ $F(X): Y$ is bounded $\}, P_{\mathrm{cp}}(X)=\{Y \in F(X): Y$ is compact $\}$, and $P_{\mathrm{cp}, \mathrm{c}}(X)=\{Y \in F(X)$ : $Y$ is compact and convex . A multivalued map $G: X \rightarrow F(X)$ is convex (closed)-valued if $G(x)$ is convex (closed) for all $x \in X$. A map $G$ is bounded on bounded sets if $G(\mathbb{B})=$ $\bigcup_{x \in \mathbb{B}} G(x)$ is bounded in $X$ for all $\mathbb{B} \in P_{\mathrm{b}}(X)$ (i.e., $\left.\sup _{x \in \mathbb{B}}\{\sup \{|y|: y \in G(x)\}\}<\infty\right)$. A map $G$ is called upper semicontinuous (u.s.c.) on $X$ if for each $x_{0} \in X$, the set $G\left(x_{0}\right)$ is a nonempty closed subset of $X$ and if for each open set $N$ of $X$ containing $G\left(x_{0}\right)$, there exists an open neighborhood $\mathcal{N}_{0}$ of $x_{0}$ such that $G\left(\mathcal{N}_{0}\right) \subseteq N$; $G$ is said to be completely continuous if $G(\mathbb{B})$ is relatively compact for every $\mathbb{B} \in P_{\mathrm{b}}(X)$. If a multivalued map $G$ is completely continuous with nonempty compact values, then $G$ is u.s.c. if and only if $G$ has a closed graph, that is, $x_{n} \rightarrow x_{*}, y_{n} \rightarrow y_{*}, y_{n} \in G\left(x_{n}\right)$ imply $y_{*} \in G\left(x_{*}\right)$. A map $G$ has a fixed point if there is $x \in X$ such that $x \in G(x)$. The fixed point set of a multivalued operator $G$ will be denoted by Fix $G$. A multivalued map $G:[0 ; 1] \rightarrow \mathcal{P}_{\mathrm{cl}}(\mathbb{R})$ is said to be measurable if for every $y \in \mathbb{R}$, the function $t \mapsto d(y, G(t))=\inf \{|y-z|: z \in G(t)\}$ is measurable.

Definition 4.1 A function $x \in A C^{n}([0,1], \mathbb{R})$ satisfying the conditions $x(0)+x^{\prime}(0)=h(x)$, $\int_{0}^{\eta} x(t) d t=\xi, x^{\prime \prime}(0)=x^{\prime \prime \prime}(0)=\cdots=x^{(n-1)}(0)=0$ is said to be a solution of problem (1.2) if there exists a function $f \in L^{1}([0,1], \mathbb{R})$ such that $f(t) \in F\left(t, x(t),{ }^{\mathrm{c}} D^{\beta} x(t)\right)$ a.e. on $[0,1]$ and

$$
\begin{aligned}
x(t)= & \int_{0}^{t} \frac{(t-s)^{\alpha-1}}{\Gamma(\alpha)} f(s) d s+\frac{2(1-t)}{\eta(2-\eta)} \xi+\frac{2 t-\eta}{2-\eta} h(x) \\
& +\frac{2(t-1)}{\eta(2-\eta)} \int_{0}^{\eta} \frac{(\eta-s)^{\alpha}}{\Gamma(\alpha+1)} f(s) d s .
\end{aligned}
$$

Definition 4.2 A multivalued map $F:[0,1] \times \mathbb{R} \times \mathbb{R} \rightarrow \mathcal{P}(\mathbb{R})$ is said to be a Carathéodory function if 
(i) $t \mapsto F(t, x, y)$ is measurable for each $x, y \in \mathbb{R}$;

(ii) $(x, y) \mapsto F(t, x, y)$ is upper semicontinuous for almost all $t \in[0,1]$.

Further, a Carathéodory function $F$ is called $L^{1}$-Carathéodory if

(iii) for each $a>0$, there exists $\varphi_{a} \in L^{1}\left([0,1], \mathbb{R}^{+}\right)$such that

$$
\|F(t, x, y)\|=\sup \{|v|: v \in F(t, x, y)\} \leq \varphi_{a}(t)
$$

for all $\|x\|,\|y\| \leq a$ and for a.e. $t \in[0,1]$.

For each $y \in C([0,1], \mathbb{R})$, define the set of selections of $F$ by

$$
S_{F, y}:=\left\{v \in L^{1}([0,1], \mathbb{R}): v(t) \in F\left(t, y(t),{ }^{\mathrm{c}} D^{\beta} y(t)\right) \text { for a.e. } t \in[0,1]\right\} .
$$

The following lemma will be used in the sequel.

Lemma 4.1 [35] Let $X$ be a Banach space. Let $F:[0,1] \times X \times X \rightarrow \mathcal{P}_{\mathrm{cp}, \mathrm{c}}(X)$ be an $L^{1}$-Carathéodory multivalued map, and let $\Theta$ be a linear continuous mapping from $L^{1}([0,1], X)$ to $C([0,1], X)$. Then the operator

$$
\Theta \circ S_{F}: C([0,1], X) \rightarrow \mathcal{P}_{\text {cp, }}(C([0,1], X)), \quad x \mapsto\left(\Theta \circ S_{F}\right)(x)=\Theta\left(S_{F, x}\right),
$$

is a closed graph operator in $C([0,1], X) \times C([0,1], X)$.

To prove our main result in this section, we use the following form of the nonlinear alternative for contractive maps ([36], Corollary 3.8).

Theorem 4.1 Let $X$ be a Banach space, and $\mathcal{D}$ a bounded neighborhood of $0 \in X$. Let $Z_{1}: X \rightarrow \mathcal{P}_{\mathrm{cp}, \mathrm{c}}(X)$ and $Z_{2}: \overline{\mathcal{D}} \rightarrow \mathcal{P}_{\mathrm{cp}, \mathrm{c}}(X)$ be two multivalued operators satisfying
(a) $Z_{1}$ is contraction, and
(b) $Z_{2}$ is u.s.c. and compact.
Then, if $G=Z_{1}+Z_{2}$, then either
(i) $G$ has a fixed point in $\overline{\mathcal{D}}$, or
(ii) there are a point $u \in \partial \mathcal{D}$ and $\lambda \in(0,1)$ with $u \in \lambda G(u)$.

Theorem 4.2 Assume that $\left(\mathrm{A}_{1}\right)$ and $\left(\mathrm{A}_{2}\right)$ hold. In addition, we suppose that:

$\left(\mathrm{H}_{1}\right) \quad F:[0,1] \times \mathbb{R} \times \mathbb{R} \rightarrow \mathcal{P}_{\mathrm{cp}, \mathrm{c}}(\mathbb{R})$ is an $L^{1}$-Carathéodory multivalued map;

$\left(\mathrm{H}_{2}\right)$ there exist continuous nondecreasing functions $\psi_{i}:[0, \infty) \rightarrow(0, \infty), i=1,2$, and a function $p \in C\left([0,1], \mathbb{R}^{+}\right)$such that

$$
\|F(t, x, y)\|_{\mathcal{P}}:=\sup \{|v|: v \in F(t, x, y)\} \leq p(t)\left[\psi_{1}(|x|)+\psi_{2}(|y|)\right]
$$

for each $(t, x, y) \in[0,1] \times \mathbb{R} \times \mathbb{R}$

$\left(\mathrm{H}_{3}\right)$ there exists a number $M>0$ such that

$$
\frac{\left(1-\ell \Lambda_{3}\right) M}{\Lambda_{1}\|p\|\left[\psi_{1}(M)+\psi_{2}(M)\right]+\Lambda_{2}}>1, \quad \ell \Lambda_{3}<1,
$$

where $\Lambda_{i}, i=1,2,3$, are defined in (3.4) and (3.5).

Then the boundary value problem (1.1) has at least one solution on $[0,1]$. 
Proof To transform problem (1.1) into a fixed point problem, we introduce the operator $\mathcal{N}: X \rightarrow \mathcal{P}(X)$ as follows:

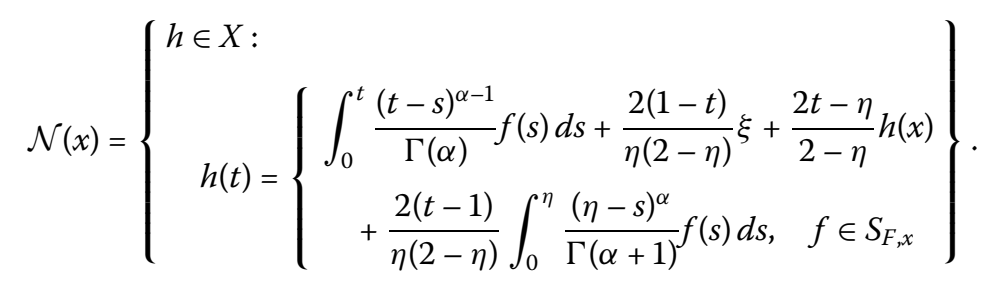

Now, we define the operator $\mathcal{A}_{1}: X \rightarrow X$ by

$$
\mathcal{A}_{1} x(t)=\frac{2(1-t)}{\eta(2-\eta)} \xi+\frac{2 t-\eta}{2-\eta} h(x)
$$

and the multivalued operator $\mathcal{A}_{2}: X \rightarrow \mathcal{P}(X)$ by

$$
\mathcal{A}_{2}(x)=\left\{\begin{array}{l}
h \in C([0,1], \mathbb{R}): \\
h(t)=\left\{\int_{0}^{t} \frac{(t-s)^{\alpha-1}}{\Gamma(\alpha)} f(s) d s+\frac{2(t-1)}{\eta(2-\eta)} \int_{0}^{\eta} \frac{(\eta-s)^{\alpha}}{\Gamma(\alpha+1)} f(s) d s\right.
\end{array}\right\} .
$$

Observe that $\mathcal{N}=\mathcal{A}_{1}+\mathcal{A}_{2}$. We shall show that the operators $\mathcal{A}_{1}$ and $\mathcal{A}_{2}$ satisfy all the conditions of Theorem 4.1 on $[0,1]$. Also, we establish that the operators $\mathcal{A}_{1}$ and $\mathcal{A}_{2}$ are such that $\mathcal{A}_{1}, \mathcal{A}_{2}: B_{r} \rightarrow \mathcal{P}_{\mathrm{cp}, \mathrm{c}}(X)$, where $B_{r}=\left\{x \in X:\|x\|_{X} \leq r\right\}$ is a bounded set in $C([0,1], \mathbb{R})$. First, we prove that $\mathcal{A}_{2}$ is compact-valued on $B_{r}$. Note that the operator $\mathcal{A}_{2}$ is equivalent to the composition $\mathcal{L} \circ S_{F}$, where $\mathcal{L}$ is the continuous linear operator from $L^{1}([0,1], \mathbb{R})$ into $X$ defined by

$$
\mathcal{L}(v)(t)=\int_{0}^{t} \frac{(t-s)^{\alpha-1}}{\Gamma(\alpha)} v(s) d s+\frac{2(t-1)}{\eta(2-\eta)} \int_{0}^{\eta} \frac{(\eta-s)^{\alpha}}{\Gamma(\alpha+1)} v(s) d s .
$$

Suppose that $x \in B_{r}$ is arbitrary and let $\left\{v_{n}\right\}$ be a sequence in $S_{F, x}$. Then, by the definition of $S_{F, x}$, we have $v_{n}(t) \in F\left(t, x(t),{ }^{\mathrm{c}} D^{\beta} x(t)\right)$ for almost all $t \in[0,1]$. Since $F\left(t, x(t),{ }^{\mathrm{c}} D^{\beta} x(t)\right)$ is compact for all $t \in J$, there is a convergent subsequence of $\left\{v_{n}(t)\right\}$ (we denote it by $\left\{v_{n}(t)\right\}$ again) that converges in measure to some $v(t) \in S_{F, x}$ for almost all $t \in J$. On the other hand, $\mathcal{L}$ is continuous, so $\mathcal{L}\left(v_{n}\right)(t) \rightarrow \mathcal{L}(v)(t)$ pointwise on $[0,1]$.

In order to show that the convergence is uniform, we have to show that $\left\{\mathcal{L}\left(v_{n}\right)\right\}$ is an equicontinuous sequence. Let $t_{1}, t_{2} \in[0,1]$ with $t_{1}<t_{2}$. Then, we have

$$
\begin{aligned}
\left|\mathcal{L}\left(v_{n}\right)\left(t_{2}\right)-\mathcal{L}\left(v_{n}\right)\left(t_{1}\right)\right| \leq & \mid \frac{1}{\Gamma(\alpha)} \int_{0}^{t_{1}}\left[\left(t_{2}-s\right)^{\alpha-1}-\left(t_{1}-s\right)^{\alpha-1}\right] v_{n}(s) d s \\
& +\frac{1}{\Gamma(\alpha)} \int_{t_{1}}^{t_{2}}\left(t_{2}-s\right)^{\alpha-1} v_{n}(s) d s \mid \\
& +\frac{2\left(t_{2}-t_{1}\right)}{\eta(2-\eta)} \int_{0}^{\eta} \frac{(\eta-s)^{\alpha}}{\Gamma(\alpha+1)}\left|v_{n}(s)\right| d s \\
\leq & {\left[\psi_{1}(r)+\psi_{2}(r)\right]\left\{\frac{1}{\Gamma(\alpha+1)}\left(t_{2}^{\alpha}-t_{1}^{\alpha}\right)+\frac{2\left(t_{2}-t_{1}\right)}{\eta \Gamma(\alpha+2)}\right\} . }
\end{aligned}
$$


Continuing this process, we have

$$
\begin{aligned}
\left|\left(\mathcal{L}^{\prime}\left(v_{n}\right)\left(t_{2}\right)\right)-\left(\mathcal{L}^{\prime}\left(v_{n}\right)\left(t_{1}\right)\right)\right| \leq & \|p\|\left[\psi_{1}(r)+\psi_{2}(r)\right]\left\{\frac{1}{\Gamma(\alpha-\beta+1)}\left(t_{2}^{\alpha-\beta-1}-t_{1}^{\alpha-\beta-1}\right)\right. \\
& \left.+\frac{2}{\eta \Gamma(\alpha+2) \Gamma(2-\beta)}\left(t_{2}^{1-\beta}-t_{1}^{1-\beta}\right)\right\} .
\end{aligned}
$$

We observe that the right-hand sides of the above inequalities tend to zero as $t_{2} \rightarrow t_{1}$. Thus, the sequence $\left\{\mathcal{L}\left(v_{n}\right)\right\}$ is equicontinuous, and by using the Arzelá-Ascoli theorem we get that there is a uniformly convergent subsequence. So, there is a subsequence of $\left\{v_{n}\right\}$ (we denote it again by $\left.\left\{v_{n}\right\}\right)$ such that $\mathcal{L}\left(v_{n}\right) \rightarrow \mathcal{L}(v)$. Note that $\mathcal{L}(v) \in \mathcal{L}\left(S_{F, x}\right)$. Hence, $\mathcal{A}_{2}(x)=\mathcal{L}\left(S_{F, x}\right)$ is compact for all $x \in B_{r}$. So $\mathcal{A}_{2}(x)$ is compact.

Now, we show that $\mathcal{A}_{2}(x)$ is convex for all $x \in X$. Let $z_{1}, z_{2} \in \mathcal{A}_{2}(x)$. We select $f_{1}, f_{2} \in S_{F, x}$ such that

$$
z_{i}(t)=\int_{0}^{t} \frac{(t-s)^{\alpha-1}}{\Gamma(\alpha)} f_{i}(s) d s+\frac{2(t-1)}{\eta(2-\eta)} \int_{0}^{\eta} \frac{(\eta-s)^{\alpha}}{\Gamma(\alpha+1)} f_{i}(s) d s, \quad i=1,2
$$

for almost all $t \in[0,1]$. Let $0 \leq \lambda \leq 1$. Then, we have

$$
\begin{aligned}
{\left[\lambda z_{1}+(1-\lambda) z_{2}\right](t)=} & \int_{0}^{t} \frac{(t-s)^{\alpha-1}}{\Gamma(\alpha)}\left[\lambda f_{1}(s)+(1-\lambda) f_{2}(s)\right] d s \\
& +\frac{2(t-1)}{\eta(2-\eta)} \int_{0}^{\eta} \frac{(\eta-s)^{\alpha}}{\Gamma(\alpha+1)}\left[\lambda f_{1}(s)+(1-\lambda) f_{2}(s)\right] d s .
\end{aligned}
$$

Since $F$ has convex values, $S_{F, u}$ is convex, and $\lambda f_{1}(s)+(1-\lambda) f_{2}(s) \in S_{F, x}$. Thus,

$$
\lambda z_{1}+(1-\lambda) z_{2} \in \mathcal{A}_{2}(x)
$$

Consequently, $\mathcal{A}_{2}$ is convex-valued. Obviously, $\mathcal{A}_{1}$ is compact and convex-valued.

The rest of the proof consists of several steps and claims.

Step 1 . We show that $\mathcal{A}_{1}$ is a contraction on $C([0,1], \mathbb{R})$. The proof is similar to that for the operator $F_{2}$ in Step 2 of Theorem 3.1.

Step 2. $\mathcal{A}_{2}$ is upper semicontinuous and compact. This will be established in several claims.

Claim I: $\mathcal{A}_{2}$ maps bounded sets into bounded sets in X. Let $B_{r}=\left\{x \in X:\|x\|_{X} \leq r\right\}$ be a bounded set in $X$. Then, for each $h \in \mathcal{A}_{2}(x), x \in B_{r}$, there exists $f \in S_{F, x}$ such that

$$
h(t)=\int_{0}^{t} \frac{(t-s)^{\alpha-1}}{\Gamma(q)} f(s) d s+\frac{2(t-1)}{\eta(2-\eta)} \int_{0}^{\eta} \frac{(\eta-s)^{\alpha}}{\Gamma(\alpha+1)} f(s) d s .
$$

Then, for $t \in[0,1]$, we have

$$
\begin{aligned}
\|h\| & \leq \int_{0}^{t} \frac{(t-s)^{\alpha-1}}{\Gamma(\alpha)}|f(s)| d s+\frac{2(t-1)}{\eta(2-\eta)} \int_{0}^{\eta} \frac{(\eta-s)^{\alpha}}{\Gamma(\alpha+1)}|f(s)| d s \\
& \leq \int_{0}^{t} \frac{(t-s)^{\alpha-1}}{\Gamma(\alpha)}|f(s)| d s+\frac{2(t-1)}{\eta(2-\eta)} \int_{0}^{1} \frac{(1-s)^{\alpha}}{\Gamma(\alpha+1)}|f(s)| d s \\
& \leq\|p\|\left[\psi_{1}(\|x\|)+\psi_{2}\left(\left\|c D^{\beta} x\right\|\right)\right]\left[\frac{1}{\Gamma(\alpha+1)}+\frac{2}{\eta \Gamma(\alpha+2)}\right]
\end{aligned}
$$




$$
\begin{aligned}
& \leq\|p\|\left[\psi_{1}\left(\|x\|_{X}\right)+\psi_{2}\left(\|x\|_{X}\right)\right]\left[\frac{1}{\Gamma(\alpha+1)}+\frac{2}{\eta \Gamma(\alpha+2)}\right] \\
& \leq\|p\|\left[\psi_{1}(r)+\psi_{2}(r)\right]\left[\frac{1}{\Gamma(\alpha+1)}+\frac{2}{\eta \Gamma(\alpha+2)}\right] .
\end{aligned}
$$

\section{Considering}

$$
h^{\prime}(t)=\int_{0}^{t} \frac{(t-s)^{\alpha-2}}{\Gamma(\alpha-1)} f(s) d s+\frac{2}{\eta(2-\eta)} \int_{0}^{\eta} \frac{(\eta-s)^{\alpha}}{\Gamma(\alpha+1)} f(s),
$$

we obtain

$$
\begin{aligned}
{ }^{\mathrm{c}} D^{\beta} h(t) & =\int_{0}^{t} \frac{(t-s)^{-\beta}}{\Gamma(1-\beta)}\left(\int_{0}^{s} \frac{(s-\tau)^{\alpha-2}}{\Gamma(\alpha-1)} f(\tau) d \tau+\frac{2}{\eta} \int_{0}^{\eta} \frac{(\eta-s)^{\alpha}}{\Gamma(\alpha+1)} f(s) d s\right) \\
& =\int_{0}^{t} \frac{(t-s)^{\alpha-1-\beta}}{\Gamma(\alpha-\beta)} f(s) d s+\frac{2 t^{1-\beta}}{\eta \Gamma(2-\beta)} \int_{0}^{\eta} \frac{(\eta-s)^{\alpha}}{\Gamma(\alpha+1)} f(s) d s,
\end{aligned}
$$

and therefore

$$
\begin{aligned}
\left\|{ }^{\mathrm{c}} D^{\beta} h\right\| \leq & \|p\|\left[\psi_{1}\left(\|x\|_{X}\right)+\psi_{2}\left(\|x\|_{X}\right)\right] \int_{0}^{t} \frac{(t-s)^{\alpha-1-\beta}}{\Gamma(\alpha-\beta)} d s \\
& +\frac{2}{\eta \Gamma(2-\beta)} \int_{0}^{1} \frac{(1-s)^{\alpha}}{\Gamma(\alpha+1)} d s \\
\leq & \|p\|\left[\psi_{1}(r)+\psi_{2}(r)\right]\left\{\frac{1}{\Gamma(\alpha-\beta+1)}+\frac{2}{\eta \Gamma(2-\beta)} \frac{1}{\Gamma(\alpha+2)}\right\} .
\end{aligned}
$$

Consequently,

$$
\begin{aligned}
\left\|\mathcal{A}_{2} x\right\|_{X}= & \left\|\mathcal{A}_{2} x\right\|+\left\|{ }^{\mathrm{c}} D^{\beta}\left(\mathcal{A}_{2} x\right)\right\| \\
\leq & \|p\|\left[\psi_{1}(r)+\psi_{2}(r)\right]\left\{\frac{1}{\Gamma(\alpha+1)}+\frac{2}{\eta \Gamma(\alpha+2)}+\frac{1}{\Gamma(\alpha-\beta+1)}\right. \\
& \left.+\frac{2}{\eta \Gamma(2-\beta)} \frac{1}{\Gamma(\alpha+2)}\right\},
\end{aligned}
$$

and thus the operator $\mathcal{A}_{2}\left(B_{r}\right)$ is uniformly bounded.

Claim II: $\mathcal{A}_{2}$ maps bounded sets into equicontinuous sets. Now let $0 \leq t_{1}<t_{2} \leq 1$. Then we have the following facts:

$$
\begin{aligned}
\left|\left(\mathcal{A}_{2} x\right)\left(t_{2}\right)-\left(\mathcal{A}_{2} x\right)\left(t_{1}\right)\right| \leq & \mid \frac{1}{\Gamma(\alpha)} \int_{0}^{t_{1}}\left[\left(t_{2}-s\right)^{\alpha-1}-\left(t_{1}-s\right)^{\alpha-1}\right] f(s) d s \\
& +\frac{1}{\Gamma(\alpha)} \int_{t_{1}}^{t_{2}}\left(t_{2}-s\right)^{\alpha-1} f(s) d s \mid \\
& +\frac{2}{\eta}\left(t_{2}-t_{1}\right) \int_{0}^{1} \frac{(1-s)^{\alpha}}{\Gamma(\alpha+1)}|f(s)| d s \\
\leq & \|p\|\left[\psi_{1}(r)+\psi_{2}(r)\right]\left\{\frac{1}{\Gamma(\alpha+1)}\left(t_{2}^{\alpha}-t_{1}^{\alpha}\right)+\frac{2\left(t_{2}-t_{1}\right)}{\eta \Gamma(\alpha+2)}\right\}
\end{aligned}
$$


and

$$
\begin{aligned}
\left|{ }^{\mathrm{c}} D^{\beta}\left(\mathcal{A}_{2} x\right)\left(t_{2}\right)-{ }^{\mathrm{c}} D^{\beta}\left(\mathcal{A}_{2}\right)\left(t_{1}\right)\right| \leq & \|p\|\left[\psi_{1}(r)+\psi_{2}(r)\right]\left\{\frac{1}{\Gamma(\alpha-\beta+1)}\left(t_{2}^{\alpha-\beta-1}-t_{1}^{\alpha-\beta-1}\right)\right. \\
& \left.+\frac{2}{\eta \Gamma(\alpha+2) \Gamma(2-\beta)}\left(t_{2}^{1-\beta}-t_{1}^{1-\beta}\right)\right\} .
\end{aligned}
$$

Hence, we have

$$
\left\|\left(\mathcal{A}_{2} x\right)\left(t_{2}\right)-\left(\mathcal{A}_{2} x\right)\left(t_{1}\right)\right\|_{X} \rightarrow 0 \quad \text { as } t_{2} \rightarrow t_{1}
$$

Thus, $\mathcal{A}_{2}$ is equicontinuous. Therefore, by the Ascoli-Arzelá theorem it follows that $\mathcal{A}_{2}: X \rightarrow \mathcal{P}(X)$ is completely continuous.

Claim III: $\mathcal{A}_{2}$ has a closed graph. Let $x_{n} \rightarrow x_{*}, h_{n} \in \mathcal{A}_{2}\left(x_{n}\right)$, and $h_{n} \rightarrow h_{*}$. Then we need to show that $h_{*} \in \mathcal{A}_{2}\left(x_{*}\right)$. Associated with $h_{n} \in \mathcal{A}_{2}\left(x_{n}\right)$, there exists $f_{n} \in S_{F, x_{n}}$ such that for each $t \in[0,1]$,

$$
h_{n}(t)=\int_{0}^{t} \frac{(t-s)^{\alpha-1}}{\Gamma(q)} f_{n}(s) d s+\frac{2(t-1)}{\eta(2-\eta)} \int_{0}^{\eta} \frac{(\eta-s)^{\alpha}}{\Gamma(\alpha+1)} f_{n}(s) d s .
$$

Thus, it suffices to show that there exists $f_{*} \in S_{F, x_{*}}$ such that for each $t \in[0,1]$,

$$
h_{*}(t)=\int_{0}^{t} \frac{(t-s)^{\alpha-1}}{\Gamma(q)} f_{*}(s) d s+\frac{2(t-1)}{\eta(2-\eta)} \int_{0}^{\eta} \frac{(\eta-s)^{\alpha}}{\Gamma(\alpha+1)} f_{*}(s) d s .
$$

Let us consider the linear operator $\Theta: L^{1}([0,1], \mathbb{R}) \rightarrow C([0,1], \mathbb{R})$ given by

$$
f \mapsto \Theta(f)(t)=\int_{0}^{t} \frac{(t-s)^{\alpha-1}}{\Gamma(q)} f(s) d s+\frac{2(t-1)}{\eta(2-\eta)} \int_{0}^{\eta} \frac{(\eta-s)^{\alpha}}{\Gamma(\alpha+1)} f(s) d s .
$$

Observe that

$$
\begin{aligned}
& \left\|h_{n}(t)-h_{*}(t)\right\| \\
& \quad=\left\|\int_{0}^{t} \frac{(t-s)^{q-1}}{\Gamma(q)}\left(f_{n}(s)-f_{*}(s)\right) d s+\frac{2(t-1)}{\eta(2-\eta)} \int_{0}^{\eta} \frac{(\eta-s)^{\alpha}}{\Gamma(\alpha+1)}\left(f_{n}(s)-f_{*}(s)\right) d s\right\| \rightarrow 0
\end{aligned}
$$

as $n \rightarrow \infty$.

Thus, it follows by Lemma 4.1 that $\Theta \circ S_{F}$ is a closed graph operator. Further, we have $h_{n}(t) \in \Theta\left(S_{F, x_{n}}\right)$. Since $x_{n} \rightarrow x_{*}$, we have

$$
h_{*}(t)=\int_{0}^{t} \frac{(t-s)^{\alpha-1}}{\Gamma(q)} f_{*}(s) d s+\frac{2(t-1)}{\eta(2-\eta)} \int_{0}^{\eta} \frac{(\eta-s)^{\alpha}}{\Gamma(\alpha+1)} f_{*}(s) d s
$$

for some $f_{*} \in S_{F, x_{*}}$. Hence, $\mathcal{A}_{2}$ has a closed graph (and therefore has closed values). In consequence, the operator $\mathcal{A}_{2}$ is upper semicontinuous.

Thus, the operators $\mathcal{A}_{1}$ and $\mathcal{A}_{2}$ satisfy all the conditions of Theorem 4.1, and hence its conclusion implies that either condition (i) or condition (ii) holds. We show that conclusion (ii) is not possible. If $x \in \lambda \mathcal{A}_{1}(x)+\lambda \mathcal{A}_{2}(x)$ for $\lambda \in(0,1)$, then there exists $f \in S_{F, x}$ such 
that

$$
\begin{aligned}
x(t)= & \lambda \int_{0}^{t} \frac{(t-s)^{\alpha-1}}{\Gamma(\alpha)} f(s) d s+\lambda \frac{2(1-t)}{\eta(2-\eta)} \xi+\lambda \frac{2 t-\eta}{2-\eta} h(x) \\
& +\lambda \frac{2(t-1)}{\eta(2-\eta)} \int_{0}^{\eta} \frac{(\eta-s)^{\alpha}}{\Gamma(\alpha+1)} f(s) d s, \quad t \in[0,1] .
\end{aligned}
$$

Following the method of the proof of Claim I, we can obtain

$$
\begin{aligned}
\|x\| \leq & \|p\|\left[\psi_{1}\left(\|x\|_{X}\right)+\psi_{2}\left(\|x\|_{X}\right)\right]\left[\frac{1}{\Gamma(\alpha+1)}+\frac{2}{\eta \Gamma(\alpha+2)}\right] \\
& +\frac{2}{\eta}|\xi|+(2+\eta) \ell\|x\|_{X}
\end{aligned}
$$

and

$$
\begin{aligned}
\left\|{ }^{\mathrm{c}} D^{\beta} x\right\| \leq & {\left[\psi_{1}\left(\|x\|_{X}\right)+\psi_{2}\left(\|x\|_{X}\right)\right]\left\{\frac{1}{\Gamma(\alpha-\beta+1)}+\frac{2}{\eta \Gamma(2-\beta)} \frac{1}{\Gamma(\alpha+2)}\right\} } \\
& +\frac{2}{\Gamma(2-\beta)}\left(\frac{|\xi|}{\eta}+\ell\|x\|_{X}\right) .
\end{aligned}
$$

Consequently,

$$
\begin{aligned}
\|x\|_{X}= & \|x\|+\left\|{ }^{\mathrm{c}} D^{\beta} x\right\| \\
\leq & \|p\|\left[\psi_{1}\left(\|x\|_{X}\right)+\psi_{2}\left(\|x\|_{X}\right)\right]\left\{\frac{1}{\Gamma(\alpha+1)}+\frac{2}{\eta \Gamma(\alpha+2)}+\frac{1}{\Gamma(\alpha-\beta+1)}\right. \\
& \left.+\frac{2}{\eta \Gamma(2-\beta)} \frac{1}{\Gamma(\alpha+2)}\right\}+\frac{2}{\eta}|\xi|\left(1+\frac{1}{\Gamma(2-\beta)}\right) \\
& +\left(2+\eta+\frac{2}{\Gamma(2-\beta)}\right) \ell\|x\|_{X} .
\end{aligned}
$$

Thus,

$$
\|x\|_{X} \leq \Lambda_{1}\|p\|\left[\psi_{1}\left(\|x\|_{X}\right)+\psi_{2}\left(\|x\|_{X}\right)\right]+\Lambda_{2}+\ell \Lambda_{3}\|x\|_{X}
$$

If condition (ii) of Theorem 4.1 holds, then there exist $\lambda \in(0,1)$ and $x \in \partial B_{r}$ with $x=$ $\lambda \mathcal{N}(x)$. Then, $x$ is a solution of (1.1) with $\|x\|_{X}=M$. Now, by inequality (4.5) we get

$$
\frac{\left(1-\ell \Lambda_{3}\right) M}{\Lambda_{1}\|p\|\left[\psi_{1}(M)+\psi_{2}(M)\right]+\Lambda_{2}} \leq 1,
$$

which contradicts (4.2). Hence, $\mathcal{N}$ has a fixed point in $[0,1]$ by Theorem 4.1 , and consequently problem (1.1) has a solution. This completes the proof.

Example 4.1 Consider the fractional differential inclusion

$$
{ }^{\mathrm{c}} D^{5 / 2} x(t) \in F\left(t, x(t),{ }^{\mathrm{c}} D^{3 / 4} x(t)\right)
$$


supplemented with the boundary conditions (3.8), where

$$
\begin{aligned}
F\left(t, x(t),{ }^{\mathrm{c}} D^{3 / 4} x(t)\right)= & {\left[\frac{1}{\sqrt{225+t}}\left(x(t)+\tan ^{-1}\left({ }^{\mathrm{c}} D^{3 / 4} x(t)\right)+\frac{\pi}{2}\right),\right.} \\
& \left.\frac{1}{4} \sin x(t)+\frac{{ }^{\mathrm{c}} D^{3 / 4} x(t)}{9\left(1+{ }^{\mathrm{c}} D^{3 / 4} x(t)\right)}+\frac{1}{10}\right] .
\end{aligned}
$$

\section{Clearly,}

$$
\left|F\left(t, x,{ }^{\mathrm{c}} D^{3 / 4} x(t)\right)\right| \leq p(t)\left(\psi_{1}(|x|)+\psi_{2}\left(\left|{ }^{\mathrm{c}} D^{3 / 4} x(t)\right|\right)\right)
$$

where $p(t)=1 / \sqrt{225+t}, \psi_{1}(|x|)=|x|, \psi_{2}(|x|)=\pi$. Using the data of Example 3.1 and condition $\left(\mathrm{H}_{3}\right)$, we find that $M>M_{1} \simeq 6.272351$. Hence, the hypothesis of Theorem 4.2 is satisfied, which implies that the fractional differential inclusion (4.6) together with (3.8) has a solution on $[0,1]$.

\section{Competing interests}

The authors declare that they have no competing interests.

\section{Authors' contributions}

Each of the authors, BA, SKN, RPA, and AA, contributed to each part of this work equally and read and approved the final version of the manuscript.

\section{Author details}

${ }^{1}$ Nonlinear Analysis and Applied Mathematics - Research Group (NAAM), King Abdulaziz University, P.O. Box 80203, Jeddah, 21589, Saudi Arabia. ²Department of Mathematics, University of loannina, loannina, 451 10, Greece. ${ }^{3}$ Department of Mathematics, Texas A\&M University, Kingsville, TX 78363-8202, USA.

\section{Acknowledgements}

This article was funded by the Deanship of Scientific Research (DSR) at King Abdulaziz University. The authors, therefore, acknowledge with thanks DSR for technical and financial support. The authors also thank the reviewers for their constructive remarks.

Received: 15 February 2016 Accepted: 10 March 2016 Published online: 21 March 2016

\section{References}

1. Samko, SG, Kilbas, AA, Marichev, Ol: Fractional Integrals and Derivatives: Theory and Applications. Gordon \& Breach, Yverdon (1993)

2. Marin, M: An evolutionary equation in thermoelasticity of dipolar bodies. J. Math. Phys. 40, 1391-1399 (1999)

3. Kilbas, AA, Srivastava, HM, Trujillo, Jj: Theory and Applications of Fractional Differential Equations. North-Holland Mathematics Studies, vol. 204. Elsevier, Amsterdam (2006)

4. Mainardi, F: Fractional Calculus and Waves in Linear Viscoelasticity: An Introduction to Mathematical Models. Imperial College Press, London (2010)

5. Klafter, J, Lim, SC, Metzler, R (eds.): Fractional Dynamics in Physics. World Scientific, Singapore (2011)

6. Baleanu, D, Diethelm, K, Scalas, E, Trujillo, JJ: Fractional Calculus Models and Numerical Methods. Series on Complexity, Nonlinearity and Chaos. World Scientific, Boston (2012)

7. Marin, M, Agarwal, RP, Mahmoud, SR: Nonsimple material problems addressed by the Lagrange's identity. Bound. Value Probl. 2013, 135 (2013)

8. Kisielewicz, M: Stochastic Differential Inclusions and Applications. Springer Optimization and Its Applications, vol. 80. Springer, New York (2013)

9. Ahmad, B, Alsaedi, A, Alghamdi, BS: Analytic approximation of solutions of the forced Duffing equation with integral boundary conditions. Nonlinear Anal., Real World Appl. 9, 1727-1740 (2008)

10. Čiegis, R, Bugajev, A: Numerical approximation of one model of the bacterial self-organization. Nonlinear Anal., Model. Control 17, 253-270 (2012)

11. Henderson, J, Ouahab, A: Fractional functional differential inclusions with finite delay. Nonlinear Anal. 70, 2091-2105 (2009)

12. Chang, Y-K, Nieto, JJ: Some new existence results for fractional differential inclusions with boundary conditions. Math. Comput. Model. 49, 605-609 (2009)

13. Goodrich, C: Existence and uniqueness of solutions to a fractional difference equation with nonlocal conditions. Comput. Math. Appl. 61, 191-202 (2011)

14. Bai, ZB, Sun, W: Existence and multiplicity of positive solutions for singular fractional boundary value problems. Comput. Math. Appl. 63, 1369-1381 (2012) 
15. Ahmad, B, Ntouyas, SK, Alsaedi, A: New results for boundary value problems of Hadamard-type fractional differential inclusions and integral boundary conditions. Bound. Value Probl. 2013, 275 (2013)

16. Gambo, YY, Jarad, F, Baleanu, D, Abdeljawad, T: On Caputo modification of the Hadamard fractional derivatives. Adv. Differ. Equ. 2014, 10 (2014)

17. Graef, JR, Kong, L, Wang, M: Existence and uniqueness of solutions for a fractional boundary value problem on a graph. Fract. Calc. Appl. Anal. 17, 499-510 (2014)

18. Wang, G, Liu, S, Zhang, L: Eigenvalue problem for nonlinear fractional differential equations with integral boundary conditions. Abstr. Appl. Anal. 2014, Article ID 916260 (2014)

19. Ahmad, B, Ntouyas, SK, Alsaedi, A, Alzahrani, F: New fractional-order multivalued problems with nonlocal nonlinear flux type integral boundary conditions. Bound. Value Probl. 2015, 83 (2015)

20. Graef, JR, Henderson, J, Ouahab, A: Fractional differential inclusions in the Almgren sense. Fract. Calc. Appl. Anal. $18(3), 673-686(2015)$

21. Agarwal, RP, Baleanu, D, Hedayati, V, Rezapour, S: Two fractional derivative inclusion problems via integral boundary condition. Appl. Math. Comput. 257, 205-212 (2015)

22. Baleanu, D, Rezapour, S, Salehi, S: On the existence of solutions for a fractional finite difference inclusion via three points boundary conditions. Adv. Differ. Equ. 2015, 242 (2015)

23. Ntouyas, SK, Etemad, S, Tariboon, J: Existence results for multi-term fractional differential inclusions. Adv. Differ. Equ. 2015, $140(2015)$

24. Ding, $X$, Nieto, JJ: Controllability and optimality of linear time-invariant neutral control systems with different fractional orders. Acta Math. Sci. 35, 1003-1013 (2015)

25. Ahmad, B, Ntouyas, SK, Tariboon, J: A study of mixed Hadamard and Riemann-Liouville fractional integro-differential inclusions via endpoint theory. Appl. Math. Lett. 52, 9-14 (2016)

26. Ahmad, B, Agarwal, RP, Alsaedi, A: Fractional differential equations and inclusions with semiperiodic and three-point boundary conditions. Bound. Value Probl. 2016, 28 (2016)

27. Ahmad, B, Ntouyas, SK, Alsaedi, A: Existence of solutions for fractional differential equations with nonlocal and average type integral boundary conditions. J. Appl. Math. Comput. (2015). doi:10.1007/s12190-015-0960-0

28. Su, X: Boundary value problem for a coupled system of nonlinear fractional differential equations. Appl. Math. Lett. 22, 64-69 (2009)

29. O'Regan, D: Fixed-point theory for the sum of two operators. Appl. Math. Lett. 9, 1-8 (1996)

30. Granas, A, Dugundji, J: Fixed Point Theory. Springer, New York (2005)

31. Zeidler, E: Nonlinear Functional Analysis and Its Application: Fixed Point-Theorems, vol. 1. Springer, New York (1986)

32. Sadovskii, BN: On a fixed point principle. Funct. Anal. Appl. 1, 74-76 (1967)

33. Deimling, K: Multivalued Differential Equations. de Gruyter, Berlin (1992)

34. Hu, S, Papageorgiou, N: Handbook of Multivalued Analysis: Theory, vol. I. Kluwer Academic, Dordrecht (1997)

35. Lasota, A, Opial, Z: An application of the Kakutani-Ky Fan theorem in the theory of ordinary differential equations. Bull. Acad. Pol. Sci., Sér. Sci. Math. Astron. Phys. 13, 781-786 (1965)

36. Petryshyn, WV, Fitzpatric, PM: A degree theory, fixed point theorems, and mapping theorems for multivalued noncompact maps. Trans. Am. Math. Soc. 194, 1-25 (1974)

\section{Submit your manuscript to a SpringerOpen ${ }^{\circ}$ journal and benefit from:}

- Convenient online submission

Rigorous peer review

- Immediate publication on acceptance

- Open access: articles freely available online

- High visibility within the field

- Retaining the copyright to your article 